\title{
High Prevalence of Food Intolerances Among US Internet Users
}

Authors: Claire L. Jansson-Knodell, MD ${ }^{1}$, Mattie White ${ }^{1}$, Carolyn Lockett ${ }^{1}$, Huiping

$\mathrm{Xu}, \mathrm{PhD}^{2}$, Andrea Shin, $\mathrm{MD}^{1}$

1. Division of Gastroenterology and Hepatology, Department of Medicine, Indiana University School of Medicine, Indianapolis, IN.

2. Department of Biostatistics, Indiana University School of Medicine, Indianapolis, IN.

Short title: Food Intolerance in US Adults

Acknowledgements: None.

Funding: Division of Gastroenterology and Hepatology at Indiana University. AS is supported by NIH K23DK122015.

Availability of data and materials: Study data is de-identified and stored on REDCAP.

Author Contributions: Study Concept and Design (CJK, AS), Data Management and Conduct of Study (MW), Data Interpretation (CJK, MW, AS), Administrative Support and Study Coordinator (CL), Statistical Analysis (HX), Drafting of Manuscript (CJK), Critical Revisions to Manuscript (CJK, MW, CL, HX, AS).

Conflicts of Interest Statement: Authors have no disclosures.

Consent for Publication: We consent to publication of this article.

Ethical Approval: This study was conducted according to the guidelines laid down in the Declaration of Helsinki and all procedures involving research study participants were approved by approved by the Indiana University Institutional Review Board (IRB-1811552678). Consent for study participation was implied if individuals chose to participate after review of the study information sheet.

Word Count: 1923 max 2000 not including the abstract, references, tables, figures and acknowledgements

Correspondence: Andrea Shin, M.D.; 702 Rotary Circle, Suite 225; Indianapolis, IN 46202; Phone: 317-278-0414; Fax: 317-278-9525; Email: ashin@iu.edu

This is the author's manuscript of the article published in final edited form as: 


\section{Abstract (250 words):}

Objective: Food intolerances are commonly reported and are predicted to have gastrointestinal health implications. We aimed to quantify the prevalence of food intolerances among United States (US) adults and identify culprit foods through a brief web-based survey.

Design: We invited participation in an online cross-sectional survey involving a single questionnaire. Data were summarized using percentages or medians \pm interquartile range (IQR). Participant characteristics by self-reported food intolerance were compared using the Wilcoxon rank sum test and Pearson's chi-square test. Adjusted analyses were performed using multivariable logistic regression.

Setting: The survey was internet-based via Amazon's mechanical Turk, a crowdsourcing website for the completion of requester-directed tasks.

Participants: Adults who were US-based internet users were invited ages 18-80.

Results: We collected 2133 survey responses (ages 18-79). The rate of food intolerance was $24.8 \%(95 \% \mathrm{Cl}, 23.0-26.6)$ in US adults. Younger $(\mathrm{p}<0.01)$, female $(p=0.05)$, and Asian, African American, or multiple race individuals $(p<0.01)$ predominated. Lactose intolerance was most common. Frequency of a non-lactose food intolerance was $18.1 \%(95 \% \mathrm{Cl}, 16.5-19.8)$. When categorized broadly, grains, fruit, lactose, fish, vegetables, alcohol, and nuts were most troublesome for individuals in that order.

Conclusions: Self-reported food intolerance is common in US internet users. The effect of food on gastrointestinal symptoms and avoidant behaviors deserves further attention.

Keywords: food intolerance; food; survey; lactose intolerance; prevalence 


\section{Introduction:}

Self-reported food intolerances are common. Most studies report prevalence rates ranging from $4-20 \%$ of the general population. ${ }^{1-3}$ In a multicenter European study measuring 'illness' or 'trouble' caused by ingestion of particular foods, rates of food intolerance were lower in Spain and Ireland compared to Scandinavian countries and Germany. ${ }^{2}$ Australia and Mexico also had higher rates of food hypersensitivity measured at $19.1 \%$ and $30.1 \%$ respectively. ${ }^{2,4}$ Prior data from the United States (US) collected over 25 years ago found a prevalence of $12 \%$ among 555 subjects. $^{2}$ There appears to be some variability across countries. The issue of food intolerance is not a novel ailment, but there remains a paucity of recent USbased research in this area.

The literature is more robust when it comes to prevalence of traditional immune-mediated IgE-associated food allergies. The pathogenesis of food intolerance or non-allergic food hypersensitivity is less clearly defined. Food chemical reactions, enzyme and transport defects, microbiome diversity and composition, perturbations the microbiota-gut-brain axis, and fermentable oligo-, di-, mono-saccharides and polyols are potential mechanisms that may contribute to food hypersensitivity. 5 Symptoms attributed to food intolerance vary, including complaints of the gastrointestinal tract such as flatulence, abdominal pain, bloating, and diarrhea. ${ }^{5}$ These adverse reactions typically resolve upon elimination of the culprit food from the diet and return with its reintroduction. Despite the inherent challenges that accompany clinical trials of nutrition-based interventions, a doubleblinded, placebo-controlled oral challenge is considered by some to be the criterion standard for identifying food-related intolerance; the number of clinically useful diagnostics is limited. ${ }^{5}$ 
Multiple studies have suggested that particular trigger foods are implicated in generating the symptoms of common gastrointestinal disorders such as irritable bowel syndrome (IBS) in as high as $84 \%$ of patients. ${ }^{7,8}$ Many IBS patients have symptoms that worsen after a meal (63\%) and exclude or limit foods from their diet $(62 \%)^{9}, 10$ Patients with functional gastrointestinal disorders exhibit higher rates of self-reported food allergies and food sensitivities that worsen abdominal symptoms than those with organic disease (OR 4.14, OR 3.08). ${ }^{11}$ The manifestations of foodrelated IBS symptoms are associated with a heavier disease burden and reduced quality of life. ${ }^{7}$ Many patients want to learn more about foods to avoid. ${ }^{12}$ Additionally, dietary restrictions and interventions have been shown to improve symptoms for some IBS patients. ${ }^{13-16}$

Given the clinical importance of this issue and the resurgence of interest in food-related mechanisms of gastrointestinal symptoms and the impact of these symptoms on health-related quality of life, we aimed to update the data on this topic in US adults. The primary aim of our study was to quantify the prevalence of selfreported food intolerances among US adults with a secondary aim to identify common culprit foods. We hypothesized that food intolerances would be common and found in a greater percentage of the population than predicted by earlier US studies. $^{2}$

\section{Methods:}

We conducted a cross-sectional study of individuals enrolled in Amazon's Mechanical Turk (MTurk), a crowdsourcing website for the completion of requesterdirected tasks. Individuals were invited to complete an online survey involving a single questionnaire on "food-related health." MTurk has been shown to approximate 
the demographics of internet users in America. ${ }^{17}$ Inclusion criteria were adults ages 18-80, a US-based internet protocol address, and MTurk approval rating $>95 \%$.

Workers with high approval ratings answer quality control questions more accurately and thus reduce response bias. ${ }^{18}$ Surveys were administered from July to August 2019. Data were collected on self-reported food intolerances and basic demographic characteristics in order to estimate the prevalence of food intolerance and to identify the most commonly implicated foods - Supplement. Survey participants received a small monetary sum $(\$ 0.10)$ upon survey completion. This study was approved by the Indiana University Institutional Review Board and consent was implied if individuals chose to participate after reviewing the study information and survey invitation.

Data were summarized using percentages or medians \pm interquartile range (IQR). Univariate comparisons between participants with and without self-reported food intolerances were performed using the Wilcoxon rank sum test for age and Pearson's chi-square test for other variables. Sample size estimates were calculated to evaluate the association between a binary variable and food intolerance. A target sample size of 875 respondents provided $80 \%$ power at the $5 \%$ significance level to detect a 2.3 odds ratio of reporting food reporting food intolerance for binary variables with a prevalence of $20 \%$, assuming the prevalence of food intolerance to be $20 \%$ in the study population, as estimated from prior reports in the literature. Adjusted associations of participant characteristics with food intolerance were assessed using multivariable logistic regression. Since age is a continuous variable, the association between age and food intolerance was examined using the LOWESS smooth scatter plot. This plot suggested a piecewise linear effect of age, where the odds of food intolerance decreased at a different rate for subjects in the 35-55 years 
age bracket compared to those in the $18-35$ years or $55+$ years age brackets. The effect of age was similar in those 18-35 years and $55+$ years. This difference was accommodated using the logistic regression with the piecewise linear effect of age in which there were two variables for age: $18-35$ years and $55+$ years or $35-55$ years. Analyses were conducted with SAS, version 9.4 (SAS Institute Inc., Cary, NC). Twosided hypothesis tests were utilized with a $p$-value of $<0.05$ considered to be statistically significant.

\section{Results:}

A total of 2200 unique surveys were received. After excluding those with an invalid response to the quality control question $(n=64)$ and ineligible workers $(n=3)$, we analyzed 2133 valid survey responses. We found that $24.8 \%(n=529 ; 95 \% \mathrm{Cl}$, 23.0-26.6\%) of US adults reported having a food intolerance and $75.2 \%(n=1604)$ did not. There were significant differences in age, sex, race, and number of prescription medications between those with and without self-reported food intolerance-Table 1 . Those with a food intolerance were younger $(p<0.01)$; more likely to be female $(p=0.05)$; and more frequently African American, Asian, or multiple races $(p<0.01)$ than those without a food intolerance. The number of prescription medications differed between those with and without a food intolerance $(p<0.01)$. After adjusting for all other covariates, food intolerance was significantly associated with younger age for those aged 18-35 and 55+ years (18-35 and 55+ OR 0.70; 95\% Cl 0.56-0.89; $\mathrm{p}<0.01$ ), but was not associated with age in those aged $35-55$ years. Adjusted analyses also revealed associations of food intolerance with race (African American OR 1.90; 95\% Cl 1.37-2.65; $p<0.01$ and Asian OR 1.76; 95\% Cl 1.19-2.61; $p<0.01$ ) and higher number of prescription medications (1-2 medications OR 1.53;95\% Cl 
1.22-1.91; $p<0.01$ and 3 or more OR 1.48; 95\% Cl $1.09-2.01 ; p=0.01$ ) - Table 2. Region was not associated with food intolerance.

The most commonly reported food intolerances were lactose, wheat, shellfish, eggs, and soy among others - Figure 1 . When categorized broadly during data analysis after survey completion, grains, fruit, lactose, fish, vegetables, alcohol, and nuts were most troublesome for individuals in that order. The prevalence of specific food intolerances in the study sample of US adults was $6.7 \%(95 \% \mathrm{Cl}, 5.6-7.7)$ for lactose, $4.8 \%(95 \% \mathrm{Cl}, 3.9-5.7)$ for wheat, $3.3 \%$ for shellfish $(95 \% \mathrm{Cl}, 2.6-4.1), 2.9 \%$ (95\% Cl, 2.2-3.6) for eggs, and $2.5 \%$ for soy $(95 \% \mathrm{Cl}, 1.9-3.2)$. Lactose was often observed in combination with another intolerance $(30.2 \%$ [ $n=43]$ of all lactose intolerance subjects [ $n=142]$ reported lactose intolerance in combination with another food); the frequency of a non-lactose food intolerance was $18.1 \%(95 \% \mathrm{Cl}, 16.5$ 19.8).

\section{Discussion:}

In our study, the prevalence of self-reported food intolerance among USbased internet-user adults was $24.8 \%$. Over a quarter of those with a food intolerance listed lactose as the culprit food; there were many individuals who acknowledged an intolerance to milk or dairy either with or without other food intolerances. Higher rates of food intolerance were reported in younger individuals, females, non-Caucasians, and in respondents with increased prescription medication-use. Not surprisingly we found lactose intolerance was common as a majority of individuals are lactase non-persisters more so in minority ethnic groups, but not all who produce less of the enzyme manifest symptoms as dose, dilution, gut transit, colonic flora, and sensitivity to fermentation products all play a role in 
symptom manifestation or lack thereof. It is notable that the lactose intolerance rate in our study was found to be lower than some reports of prevalence, which may have been due to the survey format requiring lactose-containing foods to be written in as a response.

The strengths of this study include its large sample size, use of quality control methods, and diverse geographic coverage. Participant age ranged from 18-79 years. More than $25 \%$ of the population was non-Caucasian and $9.1 \%$ were Hispanic. All 50 states and the District of Columbia were represented.

Study limitations include its survey-based nature. This design assessed selfreporting of food intolerance which could not be confirmed by a health professional. The self-reported paradigm employed in our study means that selection bias is a concern, which could manifest as over-reporting of food intolerance. Additionally, no formal definition for food intolerance was provided so results may encompass nongastrointestinal adverse reactions to foods such as anaphylaxis, oral allergy syndrome, and others. However, the primary study objective was to estimate the prevalence of perceived food intolerance as defined by the individual among a large nationwide sample of US-based adults. This sample may not be fully representative of the US population as we had many respondents between the late 20 s to early 40 s. While this may capture a common demographic for internet users, this data may not be generalizable to the population as a whole, but a majority of US adults have internet access and mTurk has been shown to be at least as representative of the US population as traditional subject pools. Our survey may have captured individuals diagnosed with irritable bowel syndrome, as the rate in the US is approximately 5$9.9 \%$, as well as healthy individuals in the general public. ${ }^{19}$ Additionally, we asked both about foods and food components (high-fructose corn syrup) or cooking 
methods (fried foods) which may have provided information on food preparation and ingredients as well as specific foods themselves, but this mix of items is often found on food-related questionnaires in the literature.

The questions asked and answers received are useful in informing future survey development. In designing a more detailed survey, it will be important to ask participants what the term food intolerance means to them and the specific nature of their symptoms. It may be important to define the term for survey takers in order to be more specific as opposed to sensitive. Severity measurements and impact on quality of life will be key to decipher. Inquiring about reproducible reactions and those that led to restricted food intake will provide useful information. Reports of any formal medical evaluation or testing will be of interest to determine how frequently perceived food intolerance is verified or assessed by a medical professional. From a broader perspective, it may also be relevant to consider the role of a changing environment and agricultural or farming practices on the development and prevalence of food intolerance in the general population. It is our hope that this preliminary and exploratory data draws some attention to the issue of food intolerance and informs upcoming research in the area.

Our findings suggest self-perceived food intolerance is common in the US and may affect 1 in 4 adults. These perceptions may have important consequences on nutritional adequacy and could play a role in numerous health-related issues including gastrointestinal diseases and symptoms. The nature and severity of food intolerances and their associated health impact warrant further study.

\section{Max Word Count: 2000}


Table 1. Sample characteristics

\begin{tabular}{|c|c|c|c|c|}
\hline \multirow{2}{*}{$\begin{array}{l}\text { Data show } N(\%) \text { unless } \\
\text { otherwise specified }\end{array}$} & \multirow{2}{*}{$\begin{array}{c}\text { Total } \\
(n=2133)\end{array}$} & \multicolumn{2}{|c|}{ Food Intolerance } & \multirow[b]{2}{*}{ P-Value } \\
\hline & & $\begin{array}{l}\text { No }(n= \\
1604)\end{array}$ & $\begin{array}{l}\text { Yes }(n= \\
529)\end{array}$ & \\
\hline Age, median (IQR) & $34(28-44)$ & $35(28.5-44)$ & $33(27-42)$ & $<0.01$ \\
\hline Female & $1434(67.2 \%)$ & $1060(66.1 \%)$ & $374(70.7 \%)$ & 0.05 \\
\hline Race & & & & $<0.01$ \\
\hline Caucasian & $1583(74.2 \%)$ & $1229(76.6 \%)$ & $354(66.9 \%)$ & \\
\hline African American & $200(9.4 \%)$ & $133(8.3 \%)$ & $67(12.7 \%)$ & \\
\hline Asian & $174(8.2 \%)$ & $120(7.5 \%)$ & $54(10.2 \%)$ & \\
\hline Multiple Races & $93(4.4 \%)$ & $67(4.2 \%)$ & $26(4.9 \%)$ & \\
\hline Other & $52(2.4 \%)$ & $37(2.3 \%)$ & $15(2.8 \%)$ & \\
\hline Prefer not to say & $31(1.5 \%)$ & $18(1.1 \%)$ & $13(2.5 \%)$ & \\
\hline Hispanic & $194(9.1 \%)$ & $136(8.5 \%)$ & $58(11.0 \%)$ & 0.09 \\
\hline Region & & & & 0.17 \\
\hline Midwest & $465(21.8 \%)$ & $355(22.1 \%)$ & $110(20.8 \%)$ & \\
\hline Northeast & $388(18.2 \%)$ & $298(18.6 \%)$ & $90(17.0 \%)$ & \\
\hline South & $855(40.1 \%)$ & $649(40.5 \%)$ & $206(38.9 \%)$ & \\
\hline West & $425(19.9 \%)$ & $302(18.8 \%)$ & $123(23.3 \%)$ & \\
\hline Born in USA & $1963(92.0 \%)$ & $1476(92.0 \%)$ & $487(92.1 \%)$ & 0.98 \\
\hline $\begin{array}{l}\text { Number of Prescription } \\
\text { medications }\end{array}$ & & & & $<0.01$ \\
\hline 0 & $1095(51.3 \%)$ & $857(53.4 \%)$ & $238(45.0 \%)$ & \\
\hline $1-2$ & $722(33.8 \%)$ & $513(32.0 \%)$ & $209(39.5 \%)$ & \\
\hline 3 or more & $316(14.8 \%)$ & $234(14.6 \%)$ & $82(15.5 \%)$ & \\
\hline
\end{tabular}

Median and interquartile range (IQR) are reported for age due to the skewness of the distribution. P-values are derived based on the Wilcoxon rank sum test for age and the Pearson's chi-square test for other variables. 
Table 2. Adjusted associations of participant characteristics with food intolerance

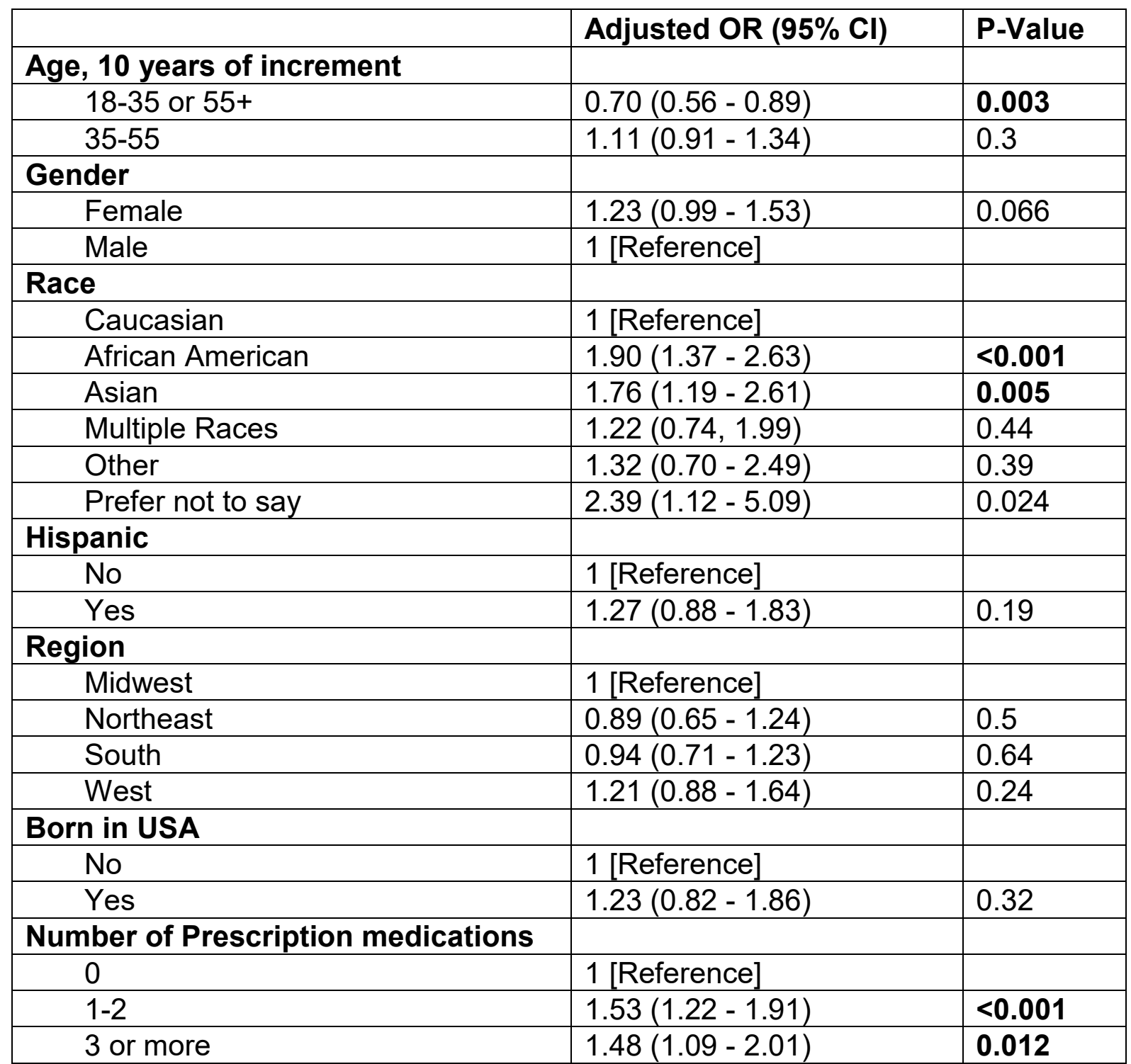

Odds ratio (OR) estimates are based on multivariable logistic regression adjusting for all other variables and assuming a piecemeal linear effect of age. 
FIGURE LEGEND

Figure 1: Food Frequency Among 529 Participants with Self-Reported Food Intolerance 


\section{References}

1. Young E, Stoneham MD, Petruckevitch A, et al. A population study of food intolerance. Lancet 1994;343:1127-30.

2. Woods RK, Abramson M, Bailey M, et al. International prevalences of reported food allergies and intolerances. Comparisons arising from the European Community Respiratory Health Survey (ECRHS) 1991-1994. Eur J Clin Nutr 2001;55:298-304.

3. Gislason D, Bjoernsson E, Gislason T. [Allergy and intolerance to food in an Icelandic urban population 20-44 years of age.]. Laeknabladid 2000;86:851-7.

4. Puente-Fernandez C, Maya-Hernandez RL, Flores-Merino MV, et al. Selfreported prevalence and risk factors associated with food hypersensitivity in Mexican young adults. Ann Allergy Asthma Immunol 2016;116:523-527 e3.

5. Lomer MC. Review article: the aetiology, diagnosis, mechanisms and clinical evidence for food intolerance. Aliment Pharmacol Ther 2015;41:262-75.

6. Chey WD. Food: The Main Course to Wellness and IIIness in Patients With Irritable Bowel Syndrome. Am J Gastroenterol 2016;111:366-71.

7. Bohn L, Storsrud S, Tornblom H, et al. Self-reported food-related gastrointestinal symptoms in IBS are common and associated with more severe symptoms and reduced quality of life. Am J Gastroenterol 2013;108:634-41.

8. Locke GR, 3rd, Zinsmeister AR, Talley NJ, et al. Risk factors for irritable bowel syndrome: role of analgesics and food sensitivities. Am J Gastroenterol 2000;95:157-65.

9. Simren M, Mansson A, Langkilde AM, et al. Food-related gastrointestinal symptoms in the irritable bowel syndrome. Digestion 2001;63:108-15.

10. Monsbakken KW, Vandvik PO, Farup PG. Perceived food intolerance in subjects with irritable bowel syndrome-- etiology, prevalence and consequences. Eur J Clin Nutr 2006;60:667-72.

11. Bhat K, Harper A, Gorard DA. Perceived food and drug allergies in functional and organic gastrointestinal disorders. Aliment Pharmacol Ther 2002;16:96973.

12. Halpert A, Dalton CB, Palsson O, et al. What patients know about irritable bowel syndrome (IBS) and what they would like to know. National Survey on Patient Educational Needs in IBS and development and validation of the Patient Educational Needs Questionnaire (PEQ). Am J Gastroenterol 2007;102:1972-82.

13. Altobelli E, Del Negro V, Angeletti PM, et al. Low-FODMAP Diet Improves Irritable Bowel Syndrome Symptoms: A Meta-Analysis. Nutrients 2017;9.

14. Halmos EP, Power VA, Shepherd SJ, et al. A diet low in FODMAPs reduces symptoms of irritable bowel syndrome. Gastroenterology 2014;146:67-75 e5.

15. Eswaran SL, Chey WD, Han-Markey T, et al. A Randomized Controlled Trial Comparing the Low FODMAP Diet vs. Modified NICE Guidelines in US Adults with IBS-D. Am J Gastroenterol 2016;111:1824-1832.

16. Staudacher HM, Lomer MCE, Farquharson FM, et al. A Diet Low in FODMAPs Reduces Symptoms in Patients With Irritable Bowel Syndrome and A Probiotic Restores Bifidobacterium Species: A Randomized Controlled Trial. Gastroenterology 2017;153:936-947.

17. Paolacci G CJ, Ipeirotis PG. Running Experiments on Amazon Mechanical Turk. Judgment and Decision Making 2010;5:411-419. 
18. Peer E, Vosgerau J, Acquisti A. Reputation as a sufficient condition for data quality on Amazon Mechanical Turk. Behav Res Methods 2014;46:1023-31.

19. Lovell RM, Ford AC. Global prevalence of and risk factors for irritable bowel syndrome: a meta-analysis. Clin Gastroenterol Hepatol 2012;10:712-721 e4. 


\section{Food Frequency Among 529 Participants With Self- Reported Food Intolerance}

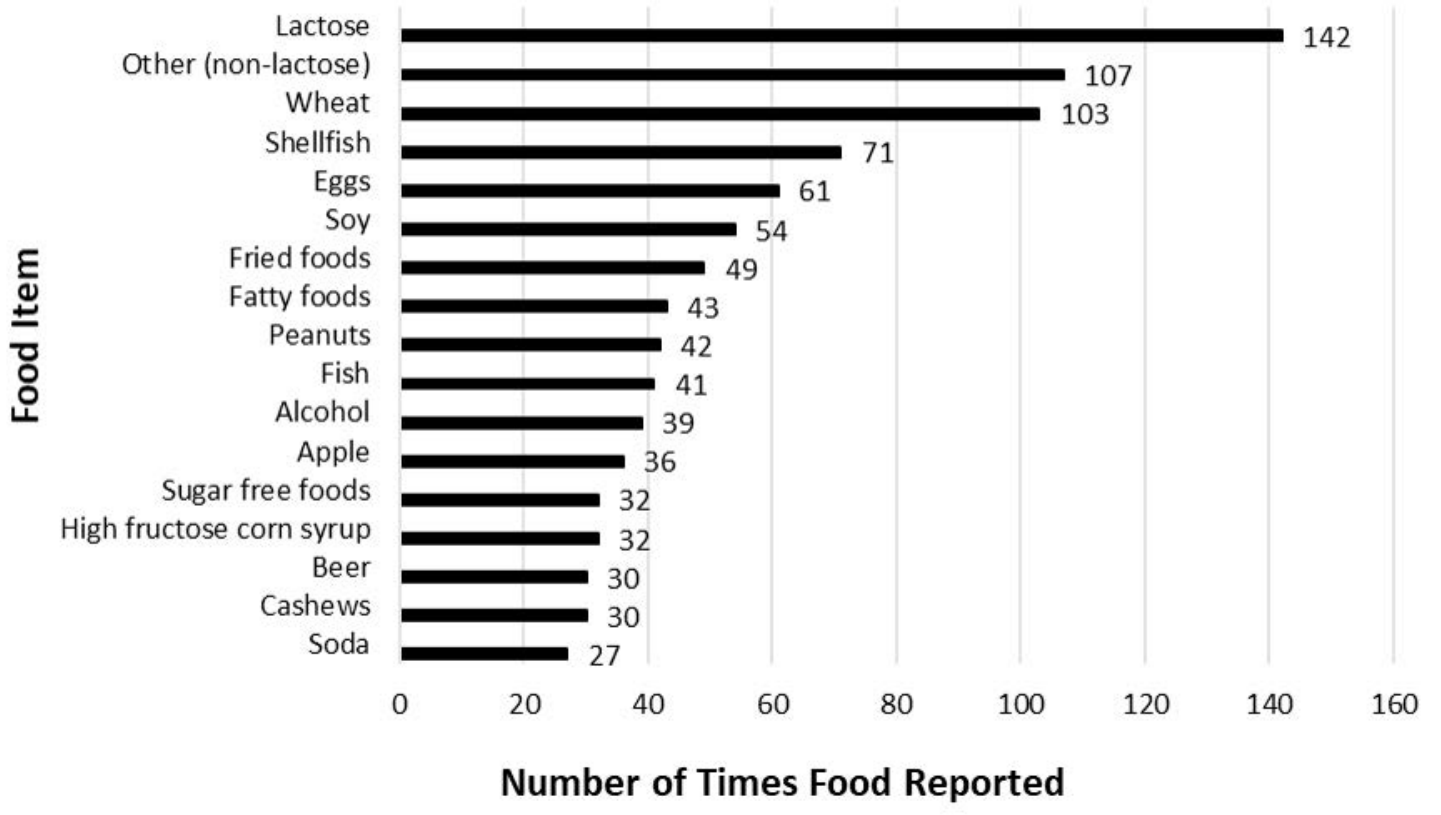




\section{SUPPLEMENT}

Survey Questions:

1. Do you have a food intolerance?

2. If so to which of the following foods?

[Wheat, protein, soy, fish, shellfish, eggs, beans, apple, nectarine, white peaches, persimmon, tamarillo, watermelon, boysenberry, figs, mango, pear, asparagus, artichokes, sugar snap peas, fruit juices, high-fructose corn syrup, honey, chicory, garlic, leek, onion, barley, rye, chickpeas, legume, lentils, pistachios, cashews, peanuts, mushrooms, mannitol, sugar-free foods, cauliflower, lychee, blackberries, apricots, flour, pasta, plum, beer, wine, salami, pork, fried foods, fatty foods, alcohol, coffee, tea, soda, other response with a blank to write-in an answer]

3. Do you take prescription medications?

4. What is your age?

5. What is your gender?

6. What is your race?

7. Are you Hispanic or Latino?

8. Which number comes after 5 and before 7 ?

9. What state do you currently live in?

10. Which continent were you born on? 